\title{
Preliminary Survey of Conidial Fungi in Weligama Mangrove Forest Indicates Higher Micro-Flora Diversity
}

\author{
Karunarathna A.V.A. ${ }^{1 *}$, Wijayawardene N.N. ${ }^{2}$, Weerahewa H.L.D. ${ }^{1}$ and Hyde K.D. ${ }^{2}$ \\ ${ }^{I}$ Department of Botany, The Open Univercity of Sri Lanka \\ ${ }^{2}$ Centre of Excellence in Fungal Research and School of Science, Mae Fah Luang University, \\ Thailand \\ *anumandrack@yahoo.com
}

\begin{abstract}
Sri Lanka is a country with rich floral and faunal diversity and listed as one of the biodiversity hotpots in the world. However, the diversity of micro-flora, including fungi is not properly documented and the existing data is out-dated. Taxonomic and diversity researches of fungi are quite a few in number compare to other South Asian and South-East Asian countries. Since, Sri Lanka is a tropical island, we determined to compare its micro-fungal diversity with other countries such as India, Malaysia, and Thailand which also have higher fungal diversity. A mangrove forest near Weligama bay in Southern Sri Lanka was selected as the first site for our preliminary survey. Micro-floral diversity in mangrove forests in Sri Lanka was not documented. For the study, samples were collected randomly. Saprobic fungi were observed from the leaf litter and wood litter. In order to observe the pathogenic fungi, leaf spots were also collected. Based on morphology, seven sexual taxa (four bitunicate and three unitunicate) and nine asexual taxa (six coelomycetous and three hyphomycetous taxa) were identified. All the collections were determined up to its generic level and compared with other species of the respective genus. Among these, six are new records from Sri Lanka. The study confirmed that, mangroves and mangrove associated flora provides preferable conditions for both sexual fungi and asexual fungi (i.e. conidial fungi, coelomycetes and hyphomycetes). It is important to carry out further works (including DNA sequence analyses) since it is important to provide taxonomic placement for conidial fungi in natural classification system.
\end{abstract}

Keywords: Conidial fungi, Coelomycetes, Hyphomycetes, Sri Lanka

Proceedings of the International Forestry and Environment Symposium 2016, Department of Forestry and Environmental Science, University of Sri Jayewardenepura, Sri Lanka. 